Präv Gesundheitsf 2013 · 8:200-203

DOI 10.1007/s11553-013-0398-x

Online publiziert: 26. Juni 2013

๑) Springer-Verlag Berlin Heidelberg 2013
Dietrich Garlichs

Geschäftsführer Deutsche Diabetes Gesellschaft, Berlin, Deutschland

\section{Das neue Präventionsgesetz der Bundesregierung}

\section{"Quantensprung" oder Wahlkampfrhetorik?}

Am 20. März 2013 hat die Bundesregierung ein Präventionsgesetz beschlossen, um Volkskrankheiten wie Diabetes und Brustkrebs einzudämmen. 2009 im Koalitionsvertrag angekündigt, wurden im vergangenen Jahr endlich „Eckpunkte für eine Präventionsstrategie“ vorgelegt, nun scheint kurz vor Ende der Legislaturperiode das Gesetzgebungsverfahren tatsächlich in Gang zu kommen.

Allein dieser Zeitablauf lässt vermuten, dass das Thema keine hohe Dringlichkeit für die Bundesregierung hat. Vielleicht schafft man es ja, das ungeliebte Vorhaben in der Hektik der auslaufenden Legislaturperiode und des beginnenden Vorwahlkampfes erfolgreich untergehen zu lassen? Oder ist es tatsächlich „ein Quantensprung für mehr Investition in Gesundheit statt in Therapie von Krankheiten", wie der CSU-Gesundheitspolitiker Singhammer das neue Gesetz feiert?

Nehmen wir mal an, es handele sich hier um den Ausdruck echten Reformwillens und nicht um symbolische Politik. Was ist das Kernstück der vorgeschlagenen Präventionsstrategie? Es geht im Wesentlichen um zwei Punkte:

- Künftig sollen Ärzte ihre Patienten verstärkt auf gesundheitliche Risiken und Belastungen untersuchen, sie beraten und ihnen entsprechende Präventionskurse empfehlen.

- Zweiter Schwerpunkt ist die betriebliche Gesundheitsförderung. Insgesamt sollen die Krankenkassen hierfür 2 EUR pro Versicherten zur Verfügung stellen.

Die Bundesregierung möchte mit dem neuen Gesetz zur Förderung der Präven- tion „das Wissen, die Befähigung und die Motivation in der Bevölkerung zu gesundheitsbewusstem Verhalten in allen Lebensphasen stärken“, heißt es in dem Entwurf. „Insbesondere sollen die Menschen zu gesundheitsbewusstem Verhalten in die Lage versetzt werden, die - wie Jugendliche mit Migrationshintergrund und Menschen mit niedrigem Bildungsstand oft schwer zu erreichen sind“. Beim Bundesgesundheitsministerium wird eine sog. Präventionskonferenz eingerichtet, die alle 4 Jahre einen Bericht über Erfolg und Misserfolg der Präventionsbemühungen erstellen soll.

Wie ist diese in 4 Jahren mühsam erarbeitete Strategie der Bundesregierung zu beurteilen? Ist sie der Problematik der modernen Lebensstilkrankheiten angemessen, wird sie relevante Effekte erzielen können? Nach allem was wir wissen, nein. Sie wird verpuffen, und die Diskussion über eine wirkungsvolle Präventionsstrategie wird in der nächsten Legislaturperiode angesichts weiter steigender chronischer Erkrankungen und finanzieller Belastungen wieder aufleben. Warum ist dem so?

\section{Kein medizinisches Problem}

Die modernen Zivilisationskrankheiten haben nicht in erster Linie einen medizinischen Ursprung. Sie sind Folge unserer veränderten westlichen Lebensweise. Wir essen doppelt so viel Fett, Zucker und Salz und halb so wenig Ballaststoffe wie uns gut täten. Wir bewegen uns zu wenig. Selbst für den Gang zum nächsten Briefkasten nehmen wir das Auto. Dagegen hat die Evolution unseren Körper so geformt, dass er, um gesund zu bleiben, mindestens 10.000 Schritte täglich braucht. Wir schaffen jedoch nur ein Drittel davon. Das hat gravierende Folgen:

- $67 \%$ der Männer und $53 \%$ der Frauen in Deutschland sind übergewichtig,

- $23 \%$ sind fettleibig (+ $23 \%$ bei Männern seit 1998),

- 6 Mio. Menschen haben Diabetes in Deutschland (+38\% seit 1998),

- auch Krebs, Herz-Kreislauf- und Atemwegserkrankungen nehmen weiter zu.

Noch in den 1950er Jahren gab es diese Lebensstilkrankheiten kaum. Weniger als $1 \%$ der erwachsenen Bevölkerung hatten damals Diabetes, heute sind es ca. $10 \%$, und Medizinprofessoren waren glücklich, wenn sie ihren Studenten einmal einen Herzinfarktpatienten vorstellen konnten.

\section{Der Appell zur Verhaltensänderung}

Die klassische Antwort der Gesundheitspolitik auf diesen „Tsunami der Lebensstilkrankheiten“ ist der Appell an die Vernunft des Einzelnen: „fdH“ (friss die Hälfte), „Trimm dich“, „Rauchen kann tödlich sein“, „Alkohol nur in Maßen“ und viele andere Mahnungen sollen uns zu gesundheitsbewusstem Verhalten anhalten. Alle diese gut gemeinten Ratschläge sind richtig, sie sind nur weitgehend wirkungslos. Wissen schützt vor Dummheit nicht. Die Politik, die vorrangig auf Information und den Aufruf zur individuellen Verhaltensänderung setzt, ist gescheitert, wie die Fakten eindrucksvoll belegen. 
Die Gründe hierfür sind relativ klar. Der individuelle Lebensstil wird geprägt in der Kindheit und den frühen Jahren, und zwar von der Familie, vom nahen sozialen Umfeld und von der "peer group". Diese grundlegende Verhaltensprägung lässt sich in späteren Jahren nur schwer verändern. Die frustrierenden Ergebnisse von Adipositastherapien oder Alkohol- und Nikotinentwöhnung machen das deutlich.

Erschwerend kommt hinzu, dass unser tägliches Umfeld einer Verhaltensänderung diametral entgegensteht. Das Essen wird zunehmend nicht mehr selbst zubereitet, findet vielfach außerhalb des Hauses statt und wird vom jederzeitigen Snacking abgelöst. $\mathrm{Zu}$ essen und zu trinken gibt es an jeder Ecke und zu jeder Zeit. Die Werbung sendet uns permanent Bilder und Botschaften von delikatem Fast Food, bequemen Convinience-Produkten und durstlöschenden Softdrinks - und das alles möglichst hochkalorisch und in XXL-Portionen.

Sich diesem Umfeld zu entziehen ist extrem schwer und soll es auch sein. Die Lebensmittelindustrie wirbt intensiv und mit den großen Idolen des Showbusiness und des Sports, die deutsche Fußballnationalmannschaft wirbt für Ferrero und läuft mit Kindern im McDonald's Tshirt aufs Spielfeld. Die Botschaft ist klar: mit solcher Art Ernährung wird man sportlich, leistungsstark und vielleicht berühmt. Es gibt Eltern, die tatsächlich glauben, dass Kinder Milch Schokolade besonders gut sei für ihre Kinder. Viele Lebensmittel werden mit irreführender Werbung verkauft, und eine effektive Nährwertkennzeichnung auf den Lebensmittelverpackungen hat die geballte Lobby der Lebensmittelindustrie erfolgreich verhindert.

Es wird alles getan, um diesen Zustand zu erhalten und zu festigen. Für Süßwaren stehen hundertmal so viele Werbegelder zur Verfügung wie für Obst- und Gemüse. Und der Versuch der Gesundheitspolitik, mit Informationen und Aufklärung durch die Bundeszentrale für gesundheitliche Aufklärung dagegenzuhalten, ist rührend. Ihr Budget erreicht nicht einmal 1\% des Budgets allein der Süßwarenwerbung.
Will die Politik die anwachsenden Lebensstilkrankheiten wirklich zurückdrängen, muss sie deren Ursachen zur Kenntnis nehmen und daran ansetzen. Sie muss versuchen einen gesunden Lebensstil in jungen Jahren zu prägen und sie muss ein flankierendes Umfeld schaffen. Sie muss anerkennen, dass die wesentlichen Faktoren außerhalb des Medizinsystems liegen, also jenseits der Kompetenzen der Gesundheitspolitik, und dass sie eine gesamtgesellschaftliche Strategie entwerfen muss, mit der bisherigen SchmalspurRessortpolitik dagegen scheitern wird.

Was wären die Kernpunkte einer gesellschaftlichen Präventionsstrategie?

In die Kindererziehung der Familie kann der Staat nicht eingreifen, und das ist gut so. Aber er kann in Kindergärten und Schulen ein entsprechendes Umfeld schaffen. Das bedeutet:

- Jeden Tag eine Stunde Sport in der Schule auf eine Weise, die den natürlichen Bewegungsdrang von Kindern fördert und die Freude daran erhält, und

- Schulessen nach klaren Qualitätsstandards (z. B. der Deutschen Gesellschaft für Ernährung) und keine gezuckerten Getränke, sondern kostenloses Trinkwasser für jeden Schüler.

Wenn wir diese einfachen Maßnahmen einführten, hätten wir sogar, wie bei der Umwelterziehung die Chance, dass Kinder die Freude an Bewegung und guter Ernährung in ihre Familien zurücktragen. Und wir setzten genau zum richtigen Zeitpunkt an. Den größten Gewichtssprung machen Kinder im Alter von 7 und 8 Jahren. Auch erreichten wir „the-difficult-to-reach“, also nicht nur die Kinder der gesundheitsbewussten Mittelschicht, sondern auch diejenigen, die auf außerschulische Informations- und Sportangebote von Krankenkassen und anderen Organisationen nicht reagieren. Wir hätten eine nachhaltige Struktur geschaffen und könnten uns von den vielfältigen Insellösungen und der Projekttites der augenblicklichen Präventionsversuche verabschieden.

\section{Verhältnisprävention}

Auch wenn wir in Kindergärten und Schulen einen gesunden Lebensstil frühzeitig prägen, bleibt das Problem, dass unser Umfeld heute das entgegengesetzte Verhalten fördert. Aus diesem Grunde vollzieht sich in der internationalen Debatte zurzeit ein Paradigmenwechsel hin zu einer stärkeren Betonung der Verhältnisprävention. Wenn der Appell an die Vernunft des Einzelnen so wenig fruchtet, dann sollten wir die Verhältnisse so gestalten, dass sie eine gesunde Lebensweise fördern, und nicht eine ungesunde. Der WHO-Slogan hierfür ist „to make the healthy choice the easier choice".

Um dies zu erreichen, empfiehlt die WHO höhere Steuern auf Tabak, Alkohol und verarbeitete Lebensmittel mit hohem Fett-, Zucker- und Salzanteil. Sie fordert zudem, die Werbung für derartige Produkte einzuschränken. Im Visier hat sie dabei insbesondere die auf Kinder zielende Vermarktung. Diese WHO Forderungen wurden in Europa im Konsens, also auch mit den Stimmen der Bundesregierung verabschiedet, auch wenn wir davon bisher wenig merken.

Mit derartigen Preissignalen könnte die Politik Signale an alle Menschen senden, nicht nur an die ohnehin gesundheitsbewussten. Gleichzeitig ließen sich die Mehreinnahmen für Maßnahmen der Gesundheitsförderung einsetzen, auch für die Verbilligung gesunder Lebensmittel.

Dass deutliche Preissignale in Verbindung mit Verbraucheraufklärung wirksam sein können, zeigt die Anti-Raucherkampagne. Der Anteil der Jugendlichen zwischen 12 und 17 Jahren, der zur Zigarette greift, hat sich in den letzten 10 Jahren mehr als halbiert. Rauchen wird unter jungen Menschen zunehmend uncool. Die Alkopops, die sehr rasch nach ihrem Aufkommen mit einer Steuer belegt wurden, sind vom Markt fast verschwunden.

In anderen Ländern wird inzwischen mit der Besteuerung gesundheitsschädlicher und adipogener Lebensmittel Ernst gemacht - über die klassischen Sondersteuern auf Tabak und Alkohol hinaus. In Deutschland haben diese Vorschläge bisher keine Befürworter in der Gesundheitspolitik. Das scheint nicht nur daran zu liegen, dass wir momentan einen libe- 
ralen Gesundheitsminister haben, sondern generell an der Angst, die Freiheit des Individuums einzuschränken. Nach dem Trauma der Diktaturen in Deutschland gibt es eine ideologische Fixierung, die nur schwer aufzubrechen ist. Trotz permanenter Misserfolge baut man weiterhin ausschließlich auf den Appell an die Vernunft des Einzelnen.

\section{Soziale Schieflage}

Das liberale Beharren auf der Verantwortung des Einzelnen ist richtig - und es ist gleichzeitig auch falsch. Natürlich ist es richtig, dem Einzelnen die Verantwortung nicht abzunehmen, aber Voraussetzung dafür ist, dass Familie oder Gesellschaft ihn dazu befähigt haben. Und hier haben wir ein entscheidendes Problem. Die modernen Lebensstilkrankheiten sind typische Krankheiten der bildungsfernen Schichten. Das Beispiel Übergewicht, Ursache vieler Lebensstilkrankheiten, zeigt das deutlich: Fettleibigkeit tritt bei Männern mit niedriger Bildung doppelt so häufig auf wie bei Männern mit hohem Schulabschluss; bei Frauen sogar 3-mal so häufig. Generell kann man sagen, je schlechter die sozioökonomische Situation, desto wahrscheinlicher ist das Auftreten von Lebensstilkrankheiten. Und das fängt früh an. In einer Stadt wie Kassel, keineswegs eine Stadt mit ausgeprägter Slum-Problematik, variiert der Anteil übergewichtiger Kinder beim Schuleintritt um das 12fache. In den wohlhabenden Stadtteilen trat Übergewicht in der Schuleingangsuntersuchung 2012 bei 2-3\% der Kinder auf, in den ärmeren Stadtteilen bei 22-23\%.

Diese soziale Problematik kumuliert in der Tatsache, dass meine Lebenserwartung in Deutschland extrem abhängig davon ist, in welche Familie ich hinein geboren werde. Die oberen $20 \%$ der Bevölkerung leben 10 Jahre länger als die unteren $20 \%$. Das ist ein gesundheitspolitischer Skandal. Und wer hier allein auf den Appell an die Verantwortung des Einzelnen baut, verkennt die Ursachen oder ist zynisch.

\section{Ressort- und Föderalismusdenken}

Wenn man mit Gesundheitspolitikern darüber spricht, dass eine wirkungsvolle Präventionsstrategie eine frühe Lebensstilprägung in Kindergarten und Schule erfordere und zweitens eine bevölkerungsweite Verhältnisprävention, erhält man häufig Zustimmung, sofern man unter vier Augen spricht. Im öffentlichen Kontext wird das schon schwieriger. Woran liegt das?

Offensichtlich traut man sich nicht den Sprung in andere Politikfelder über die Ressortgrenzen hinweg. Es gehört sich nicht, in andere Politikfelder einzugreifen. Da ist es sicherer, den Krankenkassen 2 EUR mehr pro Versicherten für Präventionsmaßnahmen zu verordnen, hier bewegt man sich auf sicherem Terrain. Und noch schwieriger scheint es zu sein, Maßnahmen zu fordern, die die Bund-Länder-Kompetenzgrenzen überspringen. Als Bundespolitiker klar und deutlich zu sagen: „Wir brauchen in jeder Schule jeden Tag eine Stunde Sport und eine Ernährung nach konkreten Qualitätskriterien“, scheint eine Sünde wider den heiligen Föderalismusgeist zu sein.

Wie mir ein Gesundheitspolitiker kürzlich sagte: „Wenn ich mehrfach mit Forderungen in die Öffentlichkeit gehe, die außerhalb meiner Kompetenz liegen, und die ich nicht verwirklichen kann, dann verbrenne ich politisch, oder ich stelle mich außerhalb des Systems und werde eine Art Peter Gauweiler“.

Wenn diese Maxime für die Mehrzahl der Politiker gilt, und dafür spricht vieles, werden Gesundheitspolitiker Ressortdenken und Föderalismusklippen nicht überwinden. Und das erklärt auch, warum, wider besseres Wissen, die neue Präventionsstrategie der Bundesregierung so zaghaft im Medizinsystem verharrt, obwohl die Ursachen für die modernen $\mathrm{Zi}$ vilisationskrankheiten außerhalb liegen.

\section{Wer könnte eine echte Präventionspolitik durchsetzen?}

Sind deshalb Hopfen und Malz verloren? Nicht unbedingt. Ressortdenken und Föderalismusklippen können unter bestimmten Umständen überwunden wer- den. Das erfordert allerdings einen massiven Druck von außen und das Wahrnehmen einer dramatischen Situation, was im allgemeinen Politikgeschäft selten der Fall ist, aber es kommt vor. In der Energiepolitik haben wir kürzlich eine drastische Politikwende vollzogen, nach einem Gau am anderen Ende der Welt. Lebensstilkrankheiten sind allerdings schleichende Krankheiten, sie haben kein dramatisches Potenzial wie die Vogelgrippe oder andere Infektionskrankheiten, und daher ist es eher unwahrscheinlich, dass eine öffentliche Betroffenheit geschaffen wird, die nötig wäre, um konventionelle Politikgrenzen zu überwinden.

Es gibt drei Strömungen außerhalb der deutschen Gesundheitspolitik, die helfen könnten, eine wirkungsvolle Prävention durchzusetzen. Hier ist einmal die internationale gesundheitspolitische Debatte zu nennen. Der Paradigmenwechsel in der internationalen Diskussion auf WHO und UN-Ebene ist oben skizziert worden. Hier wird inzwischen viel stärker auf eine Verhältnisprävention und auf klare bevölkerungsbezogene Strategien gesetzt und nicht mehr so sehr auf individuelle Maßnahmen und den Appell an die Einsichtsfähigkeit des Einzelnen. In der Folge haben mehrere europäische Länder z. B. die Besteuerung ungesunder Lebensmittel vorangetrieben, Frankreich auf Softdrinks, Finnland auf Softdrinks und Süßigkeiten, Ungarn auf Zucker und Salz. Im letzten Jahr hatte Dänemark eine Steuer auf gesättigte Fette eingeführt, sie wurde allerdings von der Nachfolgeregierung aus koalitionstaktischen Gründen aufgehoben, nicht wie manchmal behauptet wird, weil sie wirkungslos gewesen wäre, das hätte man in dieser kurzen Zeit noch gar nicht beurteilen können. Diskutiert werden ähnliche Steuern in Belgien, Großbritannien, Irland, Italien und $\mathrm{Ru}$ mänien. In den USA gibt es einen intensiven politischen Kampf um die Einführung verhältnispräventiver Maßnahmen, von der Begrenzung der Softdrinkbecher in New York auf einen halben Liter bis zu der Pflicht zur Auszeichnung der Kalorien auf Preisschildern. In Indien soll von diesem Jahr an Fast Food an den Schulen verboten werden.

Dieses sind nur einige Beispiele, die zeigen, wie weit sich die deutsche Dis- 
kussion bisher abgekoppelt hat von der internationalen. Es wird spannend sein zu beobachten, wann diese internationale Diskussion in Deutschland Früchte tragen wird, oder ob wir, wie bei der AntiRaucherkampagne, auch hier international wieder zu den Schlusslichtern gehören werden.

Wirkungsvoller als die internationale Debatte dürfte das Interesse der Finanzpolitik sein, der Explosion der Kosten durch die chronischen Krankheiten nicht länger tatenlos zuzusehen. Schon heute verursachen $20 \%$ der Versicherten $80 \%$ der Ausgaben der Krankenkassen, und das ganz überwiegend für die Behandlung chronischer Krankheiten und ihrer Folgen. Allein die Kosten der Diabetes-Epidemie betrugen 200948 Mrd. EUR, darin sind die indirekten Kosten noch nicht enthalten. Dies ist eine Steigerung von $33 \%$ gegenüber dem Jahr 2000.

Und in der Wirtschaft sieht man angesichts der demographischen Entwicklung immer klarer, wie wichtig eine frühzeitige Gesundheitsförderung ist. Die Alterung der Gesellschaft in Verbindung mit der Ausbreitung der Lebensstilkrankheiten gefährdet den Nachwuchs für eine „fitte“ Belegschaft. Zwar haben die großen Unternehmen Möglichkeiten, ihren Belegschaften eine betriebliche Gesundheitsförderung anzubieten. Aber diese kann nur begrenzt wirken, da sie für die Prägung des Lebensstils normalerweise zu spät kommt und insofern eher eine Reparaturmaßnahme für die Sekundärprävention darstellt. Außerdem ist die ganz überwiegende Zahl der Deutschen nicht in Großunternehmen beschäftigt, und für kleinere und mittlere Unternehmen ist betriebliche Gesundheitsförderung nur sehr eingeschränkt möglich.

Wie seinerzeit das preußische Militär, als es den zunehmend schlechten Gesundheitszustand seiner Rekruten erkannte, darauf drängte, Kinderarbeit zu verbieten, so wird der Wirtschaftssektor zunehmend Druck auf die Politik ausüben, Kindern und Jugendlichen die Rahmenbedingungen für ein gesundes Aufwachsen zu schaffen. Und das liegt im Interesse aller, auch derjenigen, die auf medizinische Versorgung angewiesen sind, und darauf, dass eine gute Behandlung finanzierbar bleibt. Wie Kofi Annan in diesem
Zusammenhang sagte: „To do nothing is no longer an option."

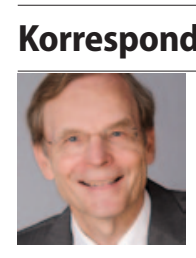

Dr. D. Garlichs

Geschäftsführer Deutsche

Diabetes Gesellschaft

Reinhardtstraße 31

10117 Berlin

garlichs@ddg.info 